\title{
PEDIATRIG RESEARGH
}

\section{General Information for Contributors}

Pediatric Research is intended to provide a medium for presentation of research communications dealing with behavioral, biochemical, physiological, and structural aspects of human development and developmental biology as well as interpretive studies leading to improved understanding of both morbid and normal processes. Research based on animal experimentation which increases comprehension of human biology or disease processes will also be considered. All manuscripts submitted will be acknowledged upon receipt and will be evaluated by at least two referees, acknowledged experts in the subject under consideration, and judged on the basis of scientific merit, timeliness, appropriateness of subject matter, and general excellence. Membership by an author in any of the three scientific societies sponsoring the Journal will in no case be a prerequisite for submission of material or acceptance of a manuscript. An attempt will be made to notify authors of the disposition of their communication in a period of less than 6 weeks from submission; unless revision or alteration is necessary, every effort will be made to publish reports within 4 months of acceptance. In general, communications will be published in the order of final acceptance after review and revision and with the understanding that they have not been published elsewhere and are not currently under review or accepted for publication in other periodicals. If and when the manuscript is published, it will become the sole property of the Journal and all copyright will be taken out in the name of the International Pediatric Research Foundation, Inc., and all rights in copyright reserved to the International Pediatric Research Foundation, Inc.

\section{Types of Communications}

Research Articles: These will document research conducted by the author in those specific scientific disciplines defined by the scope of the Journal. Manuscripts should normally be limited to approximately five thousand words, which correspond to 15-20 typescript pages. Only that illustrative material which is essential to substantiate the thesis of the communication should be submitted; the practice of submitting the same data in tabular and graphic form will be discouraged.

Research articles should be accompanied by an Extract. The technical Extract contains all of the new results reported. It contains the information, data (numbers), facts, conclusions (by reference to tables, graphs, charts, diagrams, pictures) that the author reports as novel. It omits old information and data, results of earlier research, historical background, descriptions of well-known methods and apparatus, derivations of mathematical equations, expected details of techniques, bibliography, references, acknowledgments, and the like. The technical Extract concentrates only upon new results and conclusions of the author and pares away all else.

The author should also include a one- or two-sentence statement entitled "Speculation." This will appear following the Extract and relates the observations reported to future areas of research and possible significance or correlation with other scientific research.

Reviews: These will comprise a statement of current status of a single well-defined aspect of scientific knowledge; they should provide not only a summary of the "state of the art" but in addition, illuminate the subject by combining aspects of the author's own research or thinking about the issue under discussion with germane data taken from published material. Criteria for acceptance will include incisiveness of approach. Authors should attempt to select bibliographic citations in relation to importance rather than comprehensiveness.

Letters to the Editor: While the Journal cannot be a forum for written discussion or argumentation on general or specific public or scientific issues, it will publish correspondence relating to the Journal contents. Authors of letters will be encouraged to provide in succinct form scientific criticism, rebuttals, or personal data relating to communications printed in the Journal.

\section{Page Charge Notice}

A charge of $\$ 30.00$ per page will be made for all contributed papers. This charge is an equitable share of present costs to be borne by the author and his institution. Although editorial acceptance of a manuscript is not contingent on payment of this charge, its collection is essential to the continuance of this journal. Whenever authors or their institutions indicate an inability to meet these charges they will be waived.

Complete information on manuscript preparation appears in the January issue of each volume, or may be obtained from the editorial office.

Manuscripts should be mailed to:

Charles U. Lowe, M.D.

Editor, Pediatric Research

National Institutes of Health

Building 31, Room 3A-51

Bethesda, Maryland 20014 